\title{
SOME WEAKLY COMPACT OPERATORS BETWEEN BANACH LATTICES DO NOT FACTOR THROUGH REFLEXIVE BANACH LATTICES
}

\author{
MICHEL TALAGRAND \\ ABSTRACT. We construct an example proving the claim of the title.
}

1. Introduction. A celebrated result of W. Davis, T. Figiel, W. Johnson and A. Pelczynski [2] states that a weakly compact operator between Banach spaces factors through a reflexive Banach space. It is hence natural to ask $[\mathbf{1}$, problems 1,$2 ; 2$, problems 3,4$]$ if a weakly compact operator between Banach lattices factors through a reflexive Banach lattice. The answer is known to be positive under some extra assumptions. We shall prove that it is negative in general. More precisely, we have

THEOREM A. There exists a weakly compact subset $K$ of the positive cone of $C([0,1])$ such that whenever $E$ is a reflexive Banach lattice, and $T: E \rightarrow C([0,1])$ is a bounded operator, $T\left(E_{1}\right)$ does not contain $K$ (where $E_{1}=\{x \in E ;\|x\| \leqslant 1\}$ ).

Let $\left(x_{n}\right)$ be a dense sequence in $K$. Let $U: l^{1} \rightarrow C([0,1])$ be such that $U\left(e_{n}\right)=x_{n}$, where $\left(e_{n}\right)$ denotes the canonical basis of $l^{1}$. Then $U$ is positive weakly compact. Suppose, however, that one could factor $U$ through a reflexive Banach lattice $E$ : that is, $U=T \circ V$ for $V: l^{1} \rightarrow E$ and $T: E \rightarrow C([0,1])$. Since one can suppose $\|V\| \leqslant 1$, this shows that $K \subset T\left(E_{1}\right)$, a contradiction.

The compact we shall construct is homeomorphic to $\omega^{\omega^{2}}$.

Problem. What is the smallest ordinal $\alpha$ such that there exists a weak compact set $K \subset C([0,1])$ homeomorphic to $\alpha$ that satisfies the conditions of the theorem?

ACKNOWLEDGement. My attention was brought to this problem by Professor W. Johnson.

2. Line of attack. A reflexive Banach lattice will always be represented as a space of measurable functions on a measure space $(\Omega, \Sigma, \mu)$ such that $L^{\infty}(\mu) \subset E \subset L^{1}(\mu)$ and $\|f\|_{1} \leqslant\|f\|_{E}$ for $f \in E$ [3]. Then $E^{*}$ is a space of measurable functions, and both the unit ball of $E$ and $E^{*}$ are equi-integrable.

The following technical fact is the cornerstone of the proof. It will be established in $\S 3$. We fix once and for all an ultrafilter $\mathscr{U}$ on $\mathbf{N}$.

Received by the editors October 22, 1984.

1980 Mathematics Subject Classification. Primary 46B30, 47B05; Secondary 46E30.

(C) 1986 American Mathematical Society $0002-9939 / 86 \$ 1.00+\$ .25$ per page 
MaIN FACt. Let $E$ be a reflexive Banach lattice. Suppose that we are given $b>0$, $\psi \in E_{1}^{*}$; for $n \in \mathbf{N}$, an element $\psi_{n} \in E_{1}^{*} ; \phi \in E$; for $q \in \mathbf{N}$, an element $\phi_{q}$ of $E$; and for $p \leqslant q$ and each sequence $p \leqslant i_{1}<i_{2}<\cdots<i_{p}$ of $\mathbf{N}$, an element $\phi_{q}\left(i_{1}, \ldots, i_{p}\right) \in E$ such that the following conditions hold:

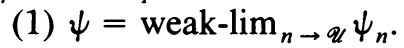

(2) For each $p<q$, each $p \leqslant i_{1}<\cdots<i_{p}$,

$$
\phi_{q}\left(i_{1}, \ldots, i_{p}\right)=\underset{\substack{i_{p+1} \rightarrow \mathscr{U} \\ \text { weak- } \lim }}{ } \phi_{q}\left(i_{1}, \ldots, i_{p}, i_{p+1}\right)
$$

(for $p=0$ this condition means $\phi_{q}=$ weak- $\lim _{i \rightarrow \mathscr{U}} \phi_{q}(i)$ ).

(3) $\phi=$ weak- $\lim _{q \rightarrow \mathscr{Q}} \phi_{q}$.

(4) There exists $A>0$ with $\left\|\phi_{q}\left(i_{1}, \ldots, i_{p}\right)\right\| \leqslant A$ whenever $p \leqslant q, p \leqslant i_{1}<\cdots$ $<i_{p}$.

(5) For each $p \leqslant q, p \leqslant i_{1}<\cdots<i_{p}<n$,

$$
\left|\int \psi_{n}\left(\phi_{q}\left(i_{1}, \ldots, i_{p}, n\right)-\phi_{q}\left(i_{1}, \ldots, i_{p}\right)\right) d \mu\right| \geqslant 1 \text {. }
$$

(6) $\int|\psi \phi| d \mu>b$.

Then there exist $p \leqslant q, p \leqslant i_{1}<\cdots<i_{p}$, and $n$ such that

$$
\int\left|\psi_{n} \phi_{q}\left(i_{1}, \ldots, i_{p}\right)\right| d \mu>b+1 .
$$

We now show how to derive the theorem from the fact. Let $\overline{\mathbf{N}}=\mathbf{N} \cup\{\infty\}$. Let

$$
L=\left\{h: \overline{\mathbf{N}} \rightarrow\{0,1\} ; \exists p \leqslant i_{1}<\cdots<i_{p} ; h(i)=0 \text { for } i \neq i_{1}, \ldots, i_{p}\right\} .
$$

Then $L \subset C(\overline{\mathbf{N}})$ is weakly compact. Let $l>0$. Consider the map $\theta_{l}$ from $L^{\prime}$ to $C\left(\overline{\mathbf{N}}^{\prime}\right)$, given by $\theta_{l}\left(h_{1}, \ldots, h_{l}\right)\left(n_{1}, \ldots, n_{l}\right)=1$ if the number of indexes $i$ for which $h_{i}\left(n_{i}\right)=1$ is even, and $\theta_{l}\left(h_{1}, \ldots, h_{l}\right)\left(n_{1}, \ldots, n_{l}\right)=0$ otherwise. The map $\theta_{l}$ is continuous when $C\left(\overline{\mathbf{N}}^{\prime}\right)$ is equipped with the pointwise convergence topology, so $K_{l}=\theta_{l}\left(L^{l}\right)$ is a weakly compact set.

For $t \in \overline{\mathbf{N}}^{l}$, we denote by $\delta_{t}$ the Dirac measure at $t$.

Proposition 1. Let $E$ be a reflexive Banach lattice, and $T: E \rightarrow C\left(\overline{\mathbf{N}}^{l}\right)$ an operator with $\|T\| \leqslant 1$. Then either $K, \not \subset T(E)$ or

$$
\sup _{x \in K_{l}} \inf _{\substack{f \in E \\ T(f)=x}} \sup _{t \in \mathbf{N}^{\prime}} \int\left|T^{*}\left(\delta_{t}\right) f\right| d \mu \geqslant l .
$$

Proof. The proof is by induction over $l$. Assume that the result holds for $l-1$ but not for $l$ (the case $l=1$ is identical to the general case). We can assume $K_{l} \subset T(E)$. There is $b<l$ such that for each $\left(h_{1}, \ldots, h_{l}\right) \in L^{l}$ there is $f\left(h_{1}, \ldots, h_{l}\right)$ in $E$ with

$$
T\left(f\left(h_{1}, \ldots, h_{l}\right)\right)=\theta_{l}\left(h_{1}, \ldots, h_{l}\right)
$$

and

$$
\forall t \in \mathbf{N}^{\prime}, \quad \int\left|T^{*}\left(\delta_{t}\right) f\left(h_{1}, \ldots, h_{l}\right)\right| d \mu<b
$$


For $p<i_{1}<\cdots<i_{p}$ we have $1_{\left\{i_{1}, \ldots, i_{p}\right\}} \in L$. Fix $q \in \mathbf{N}$. For $p \leqslant q, p<i_{1}<$ $\cdots<i_{p}$, we define $g_{q}\left(h_{1}, \ldots, h_{l-1} ; i_{1}, \ldots, i_{p}\right)$ in the following way. First we set

$$
g_{q}\left(h_{1}, \ldots, h_{l-1} ; i_{1}, \ldots, i_{q}\right)=f\left(h_{1}, \ldots, h_{l-1}, 1_{\left\{i_{1}, \ldots, i_{q}\right\}}\right) \text {. }
$$

For $0<p<q$ we define $g_{q}\left(h_{1}, \ldots, h_{l-1} ; i_{1}, \ldots, i_{p}\right)$ by decreasing induction over $p$ :

$$
g_{q}\left(h_{1}, \ldots, h_{l-1} ; i_{1}, \ldots, i_{p}\right)=\underset{\substack{i_{p+1} \rightarrow \mathscr{U} \\ \text { weak-lim }}}{ } g_{q}\left(h_{1}, \ldots, h_{l-1} ; i_{1}, \ldots, i_{p+1}\right) \text {. }
$$

For $p=0$ we set

$$
g_{q}\left(h_{1}, \ldots, h_{l-1}\right)=\underset{i \rightarrow \mathscr{U}}{\text { weak- } \lim } g_{q}\left(h_{1}, \ldots, h_{l-1} ; i\right) .
$$

Finally, we set

$$
g\left(h_{1}, \ldots, h_{l-1}\right)=\underset{q \rightarrow \mathscr{U}}{\text { weak- } \lim } g_{q}\left(h_{1}, \ldots, h_{l-1}\right) .
$$

We identify $\overline{\mathbf{N}}^{l-1}$ to $\overline{\mathbf{N}}^{l-1} \times\{\infty\} \subset \overline{\mathbf{N}}^{l}$. Let $P$ be the corresponding restriction map from $C\left(\overline{\mathbf{N}}^{l}\right)$ to $C\left(\overline{\mathbf{N}}^{l-1}\right)$. We have $\|P \circ T\| \leqslant 1$. From (7) we see that

$$
P \circ T\left(f\left(h_{1}, \ldots, h_{l}\right)\right)=P\left(\theta_{l}\left(h_{1}, \ldots, h_{l}\right)\right)=\theta_{l-1}\left(h_{1}, \ldots, h_{l-1}\right) .
$$

Since $T$ is weakly continuous, it follows by (9)-(12) that

$$
P \circ T\left(g\left(h_{1}, \ldots, h_{l-1}\right)\right)=\theta_{l-1}\left(h_{1}, \ldots, h_{l-1}\right) .
$$

Since $b-1<l-1$, the induction hypothesis gives $\left(h_{1}, \ldots, h_{l-1}\right) \in L^{l-1}$ and $t \in$ $\overline{\mathbf{N}}^{l-1}$ such that

$$
\int\left|(P \circ T)^{*}\left(\delta_{t}\right) g\left(h_{1}, \ldots, h_{l-1}\right)\right| d \mu>b-1 .
$$

If $t=\left(n_{1}, \ldots, n_{l-1}\right), t^{\prime}=\left(n_{1}, \ldots, n_{l-1}, \infty\right)$, this means that

$$
\int\left|T^{*}\left(\delta_{t^{\prime}}\right) g\left(h_{1}, \ldots, h_{l-1}\right)\right| d \mu>b-1 .
$$

Let $\psi=T^{*}\left(\delta_{t^{\prime}}\right)$. Since $T^{*}$ is weakly continuous, we have

$$
\psi=\underset{n}{\operatorname{weak}}-\lim \psi_{n},
$$

where $\psi_{n}=T^{*}\left(\delta_{\left(n_{1}, \ldots, n_{l-1}, n\right)}\right)$. Let $\phi=g\left(h_{1}, \ldots, h_{l-1}\right), \phi_{q}=g_{q}\left(h_{1}, \ldots, h_{l-1}\right)$, and for $p \leqslant q, p \leqslant i_{1}<\cdots<i_{p}$, let

$$
\phi_{q}\left(i_{1}, \ldots, i_{p}\right)=g_{q}\left(h_{1}, \ldots, h_{l-1} ; i_{1}, \ldots, i_{p}\right) .
$$

Then condition (1) follows from (14). Condition (2) follows from (10) and (11). Condition (3) follows from (13), condition (4) holds with $A=b$. Condition (6) follows from (13), and condition (5) follows from the definition of $\theta_{l}$. The hypotheses of the main fact are satisfied. So there exists $n, q$, and $p \leqslant q, p<i_{1}<\cdots<i_{p}$, such that

$$
\int\left|\psi_{n} \phi_{q}\left(i_{1}, \ldots, i_{p}\right)\right| d \mu>b
$$

Condition (15) means that

$$
\int\left|T^{*}\left(\delta_{\left(n_{1}, \ldots, n_{l-1}, n\right)}\right) g_{q}\left(h_{1}, \ldots, h_{l-1} ; i_{1}, \ldots, i_{p}\right)\right| d \mu>b .
$$


Let

$$
\xi=\operatorname{sign} T^{*}\left(\delta_{\left(n_{1} \ldots, n_{l-1}, n\right)}\right) g_{q}\left(h_{1}, \ldots, h_{l-1} ; i_{1}, \ldots, i_{p}\right)
$$

so

$$
\int \xi T^{*}\left(\delta_{\left(n_{1}, \ldots, n_{l-1}, n\right)}\right) g_{q}\left(h_{1}, \ldots, h_{l-1} ; i_{1}, \ldots, i_{p}\right) d \mu>b
$$

Conditions (9) and (11) show that one can pick (by induction) $i_{p}<i_{p+1}<\cdots<i_{q}$ such that

$$
\int \xi T^{*}\left(\delta_{\left(n_{1}, \ldots, n\right)}\right) f\left(h_{1}, \ldots, h_{l-1}, 1_{\left\{i_{1}, \ldots, i_{q}\right\}}\right) d \mu>b
$$

This contradicts (8) and finishes the proof.

We now conclude the proof of Theorem A. Denote by $M$ the Alexandroff compactification of the discrete sum of the sets $\overline{\mathbf{N}}$. Consider each $K_{\text {l }}$ as a subset of $C(M)$ by extending the functions of $K_{l}$ to zero outside $\overline{\mathbf{N}}^{\prime}$. Let $K=\bigcup K_{l}$. By construction, $K$ consists of functions taking the values zero or one, so $K$ is contained in the positive cone of $C(M)$. Proposition 1 shows that if $T$ is an operator from $E$ to $C(M)$, then for $l>\|T\|$ we have $K, \not \subset T\left(E_{1}\right)$, so $K \not \subset T\left(E_{1}\right)$. To conclude, one notices that there is a positive embedding of $C(M)$ in $C([0,1])$, such that its image is complemented.

3. Proof of the Main Fact. Let $\varepsilon=\frac{1}{8}\left(\int|\phi \psi| d \mu-b\right)$. Let $b^{\prime}=b+6 \varepsilon$, so we have $\int|\phi \psi| d \mu>b^{\prime}+\varepsilon$. Let $\xi=\operatorname{sign} \phi \psi$.

LemmA 1. $\exists a>0, \forall r, \exists q \geqslant r, \exists I \subset \mathbf{N}, I \in \mathscr{U}, \forall n \in I$,

$$
\int_{\left\{\left|\psi_{n}\right|>a\right\}}\left|\psi_{n} \phi_{q}\right| d \mu \leqslant \varepsilon ; \quad \int \xi \psi \phi_{q} d \mu>b^{\prime}
$$

Proof. Let

$$
J=\left\{q \in \mathbf{N} ; \int \xi \psi \phi_{q} d \mu>b^{\prime}\right\} .
$$

Then $J$ is infinite since $\int \xi \psi \phi d \mu>b^{\prime}$ and since $\phi$ is a weak cluster point of $\left\{\phi_{q} ; q \in J\right\}$. Let $\phi^{\prime}$ be a weak cluster point of $\left\{\left|\phi_{q}\right| ; q \in J\right\}$. Let $d>0$ be large enough that $\left\|\phi^{\prime}-\phi \wedge d\right\|_{E} \leqslant \varepsilon / 3$. Since $E_{1}^{*}$ is equi-integrable, there exists $a>0$ large enough that

$$
y \in E_{1}^{*} \Rightarrow \int_{\{|y| \geqslant a\}}|y| d \mu \leqslant \frac{\varepsilon}{3 d} .
$$

Since $\phi^{\prime}$ is a weak cluster point of $\left\{\left|\phi_{q}\right| ; q \in J ; q \geqslant r\right\}$, there exists a finite subset $J^{\prime}$ of $J$, such that $q \geqslant r$ for $q \in J^{\prime}$, and numbers $\alpha_{q} \geqslant 0, \sum_{q \in J^{\prime}} \alpha_{q}=1$, with $\left\|\sum \alpha_{q}\left|\phi_{q}\right|-\phi^{\prime}\right\|_{E} \leqslant \varepsilon / 3$. 
For each $n$ we get, with $H=\left\{\left|\psi_{n}\right| \geqslant a\right\}$,

$$
\begin{aligned}
\int_{H} \sum_{q \in J^{\prime}} \alpha_{q}\left|\phi_{q}\right|\left|\psi_{n}\right| d \mu= & \int_{H}\left(\sum \alpha_{q}\left|\phi_{q}\right|-\phi^{\prime}\right)\left|\psi_{n}\right| d \mu \\
& +\int_{H}\left(\phi^{\prime}-\phi^{\prime} \wedge d\right)\left|\psi_{n}\right| d \mu+\int_{H} \phi^{\prime} \wedge d\left|\psi_{n}\right| d \mu \\
\leqslant & \left\|\sum \alpha_{q}\left|\phi_{q}\right|-\phi^{\prime}\right\|_{E}\left\|\psi_{n}\right\|_{E^{*}}+\left\|\phi^{\prime}-\phi^{\prime} \wedge d\right\|_{E}\left\|\psi_{n}\right\|_{E^{*}} \\
& +\left\|\phi^{\prime} \wedge d\right\|_{\infty}\left\|\psi_{n} 1_{H}\right\|_{1} \leqslant \varepsilon / 3+\varepsilon / 3+d \varepsilon / 3 d \leqslant \varepsilon
\end{aligned}
$$

So for each $n$ there is $q \in J^{\prime}$ with $\int_{H}\left|\phi_{q} \psi_{n}\right| d \mu \leqslant \varepsilon$.

It follows that there is $q \in J^{\prime}$ such that the set $I=\left\{n ; \int\left|\phi_{q} \psi_{n}\right| d \mu \leqslant \varepsilon\right\}$ belongs to $\mathscr{U}$. The proof is complete.

Recall that $E$ is represented as a space of measurable functions on $(\Omega, \Sigma, \mu)$. For $q \leqslant i_{1}<i_{2}<\cdots<i_{q}$, let $\nu_{q}\left(i_{1}, \ldots, i_{q}\right)$ be the distribution on $\mathbf{R}^{q+1}$ of the function $\Omega \rightarrow \mathbf{R}^{q+1}$ given by

$$
\omega \rightarrow\left(\phi_{q}(\omega), \phi_{q}\left(i_{1}\right)(\omega), \ldots, \phi_{q}\left(i_{1}, \ldots, i_{q}\right)(\omega)\right) .
$$

If $E_{A}=\{f \in E,\|f\| \leqslant A\}$, let

$$
a_{n}=\operatorname{Sup}_{E_{A}} \int_{\{|f| \geqslant n\}}|f| d \mu
$$

Since $E_{A}$ is equi-integrable, we have $a_{n} \rightarrow 0$. Denote $x \in \mathbf{R}^{q+1}$ by $\left(x_{1}, \ldots, x_{q+1}\right)$. Let $L_{q}$ be the set of probabilities $\nu$ on $\mathbf{R}^{q+1}$ that satisfy the condition

$$
\forall n, \forall i \leqslant q+1, \quad \int_{\left\{\left|x_{i}\right| \geqslant n\right\}}\left|x_{i}\right| d \nu(x) \leqslant a_{n} .
$$

Then $L_{q}$ is weakly compact, and $\nu_{q}\left(i_{1}, \ldots, i_{q}\right) \in L_{q}$ for each $q<i_{1}<\cdots<i_{q}$. Hence, we can define

$$
\nu_{q}=\lim _{i_{1} \rightarrow \mathscr{U}} \lim _{i_{2} \rightarrow \mathscr{U}} \cdots \lim _{i_{q} \rightarrow \mathscr{U}} \nu_{p}\left(i_{1}, \ldots, i_{q}\right)
$$

where the limit is in the weak sense. We now fix $q$. For $i \leqslant q+1$ we denote by $\Sigma_{i}$ the $\sigma$-algebra on $\mathbf{R}^{q+1}$ generated by the coordinates of rank $\leqslant i$.

LEMMA 2. When $\mathbf{R}^{q+1}$ is equipped with $\nu_{q}$, the sequence $\left(x_{i}\right)_{i \leqslant q+1}$ is a martingale with respect to the filtration $\left(\Sigma_{i}\right)_{i \leqslant q+1}$.

Proof. Fix $j \leqslant q$. Let $s$ be a bounded continuous function on $\mathbf{R}^{q+1}$ that depends only on $x_{1}, \ldots, x_{j}$. For $a>0$ and for a function $f$ on $\mathbf{R}^{q+1}$ or on $\Omega$, we denote by $f^{a}$ its truncation at $a$ and $-a$ : that is, $f^{a}=(f \wedge a) \vee(-a)$. We have

$$
\begin{aligned}
\int x_{j+1} s(x) d \nu_{q}(x) & =\lim _{a \rightarrow \infty} \int x_{j+1}^{a} s(x) d \nu_{q}(x) \\
= & \lim _{a \rightarrow \infty} \lim _{i_{1} \rightarrow \mathscr{U}} \lim _{i_{2} \rightarrow \mathscr{U}} \cdots \lim _{i_{q} \rightarrow \mathscr{U}} \int \phi_{q}^{a}\left(i_{1}, \ldots, i_{j+1}\right) s\left(\phi_{q}, \ldots, \phi_{q}\left(i_{1}, \ldots, i_{j}\right)\right) .
\end{aligned}
$$


We now note that we have $\left\|f-f^{a}\right\|_{1} \rightarrow 0$ uniformly for $f \in E_{A}$, so we can intervert the limits and get

$$
\begin{aligned}
\int x_{j+1} s(x) d \nu_{q}(x) \\
\quad=\lim _{i_{1} \rightarrow \mathscr{U}} \cdots \lim _{i_{q} \rightarrow \mathscr{U}} \lim _{a \rightarrow \infty} \int \phi_{q}^{a}\left(i_{1}, \ldots, i_{j+1}\right) s\left(\phi_{q}, \ldots, \phi_{q}\left(i_{1}, \ldots, i_{j}\right)\right) d \mu \\
=\lim _{i_{1} \rightarrow \mathscr{U}} \cdots \lim _{i_{j+1} \rightarrow \mathscr{U}} \int \phi_{q}\left(i_{1}, \ldots, i_{j+1}\right) s\left(\phi_{q}, \ldots, \phi_{q}\left(i_{1}, \ldots, i_{j}\right)\right) d \mu .
\end{aligned}
$$

We now note that $s\left(\phi_{q}, \ldots, \phi_{q}\left(i_{1}, \ldots, i_{j}\right)\right) \in L^{\infty} \subset E^{*}$. Since

$$
\underset{i_{j+1} \rightarrow \mathscr{U}}{\text { weak- } \lim _{q}} \phi_{q}\left(i_{1}, \ldots, i_{j}, i_{j+1}\right)=\phi_{q}\left(i_{1}, \ldots, i_{j}\right) \text {, }
$$

we get

$$
\begin{aligned}
\int x_{j+1} s(x) d \nu_{q}(x) \\
\quad=\lim _{i_{1} \rightarrow \mathscr{U}} \cdots \lim _{i_{j} \rightarrow \mathscr{U}} \int \phi_{q}\left(i_{1}, \ldots, i_{j}\right) s\left(\phi_{q}, \phi_{q}\left(i_{1}\right), \ldots, \phi_{q}\left(i_{1}, \ldots, i_{j}\right)\right) d \mu \\
=\lim _{i_{1} \rightarrow \mathscr{U}} \cdots \lim _{i_{j} \rightarrow \mathscr{U}} \lim _{a \rightarrow \infty} \int \phi_{q}^{a}\left(i_{1}, \ldots, i_{j}\right) s\left(\phi_{q}, \phi_{q}\left(i_{1}\right), \ldots, \phi_{q}\left(i_{1}, \ldots, i_{j}\right)\right) d \mu \\
=\lim _{a \rightarrow \infty} \lim _{i_{1} \rightarrow \mathscr{U}} \ldots \lim _{i_{j} \rightarrow \mathscr{U}} \int \phi_{q}^{a}\left(i_{1}, \ldots, i_{j}\right) s\left(\phi_{q}, \ldots, \phi_{q}\left(i_{1}, \ldots, i_{j}\right)\right) d \mu \\
=\lim _{a \rightarrow \infty} \int x_{j}^{a} s\left(x_{1}, \ldots, x_{j}\right) d \nu_{q}(x) \\
=\int x_{j} s\left(x_{1}, \ldots, x_{j}\right) d \nu_{q}(x) .
\end{aligned}
$$

The lemma is proved.

The following elementary result is likely to be known; but we do not know a reference for it.

Lemma 3. Let $A>0, \eta>0$. Then there exists $N(A, \eta)$ depending only on $A$ and $\eta$ such that for any martingale $\left(X_{j}\right)_{j \leqslant q}$, with $\left\|X_{j}\right\|_{1} \leqslant A$ for $j \leqslant q$, we have

$$
\operatorname{card}\left\{j<q ; P\left(\left\{\left|X_{j+1}-X_{j}\right|>\eta\right\}\right)>\eta\right\} \leqslant N(A, \eta)
$$

Proof. Since $X_{j}$ is the difference of two positive martingales $\left(Y_{j}\right),\left(Z_{j}\right)$ for which $\left\|Y_{j}\right\|_{1},\left\|Z_{j}\right\|_{1} \leqslant A$, we can suppose that $X_{j} \geqslant 0$. Let $h=3 A / \eta$, so for each $j \leqslant q$ we have $P\left(\left\{X_{j} \geqslant h\right\}\right) \leqslant \eta / 3$. Let $Y_{j}=h-X_{j} \wedge h$. Then $0 \leqslant Y_{j} \leqslant h$, and $E^{j}\left(Y_{j+1}\right) \geqslant Y_{j}$ for $1 \leqslant j<q$. We have

$$
E\left(\left(Y_{j+1}-Y_{j}\right)^{2}\right) \leqslant E\left(Y_{j+1}^{2}\right)+E\left(Y_{j}^{2}\right)-2 E\left(Y_{j} Y_{j+1}\right) \leqslant E\left(Y_{j+1}^{2}\right)-E\left(Y_{j}^{2}\right),
$$

so $\sum_{j=1}^{q-1} E\left(Y_{j+1}-Y_{j}\right)^{2} \leqslant h^{2}$. Let

$$
A=\left\{j<q ; E\left(Y_{j+1}-Y_{j}\right)^{2} \geqslant \eta^{2} / 3\right\},
$$

so card $A \leqslant N(A, \eta)=2 h^{2} / \eta^{2}$. For $j \in A$ we have $P\left(\left\{\left|Y_{j+1}-Y_{j}\right| \geqslant \eta\right\}\right) \leqslant \eta / 3$, so $P\left(\left\{\left|X_{i+1}-X_{i}\right| \geqslant \eta\right\}\right) \leqslant \eta$. The proof is complete. 
We now prove the Main Fact.

Let $a$ be as in Lemma 1. Since the unit ball of $E$ is equi-integrable, there exists $\eta>0$ such that for $x \in E,\|x\| \leqslant 2 a$, we have

$$
\mu(\{|x|>\eta\})<\eta \Rightarrow\|x\|_{1} \leqslant \varepsilon / a .
$$

Let $N(A, \eta)$, as in Lemma 3, and let $r=N(A, \eta)+1$. Let $q \geqslant r, I$ be as in Lemma 1. We apply Lemma 3 to the martingale $\left(x_{j}\right)_{j \leqslant q+1}$ on $\left(\mathbf{R}^{q+1}, \nu_{q}\right)$, so we get $j \leqslant q$ such that $\nu_{q}\left(\left\{\left|x_{j+1}-x_{j}\right|<\eta\right\}\right)>1-\eta$.

Consider the set $T$ of families $i_{1}<\cdots<i_{j}$ such that the following set $J$ belongs to $\mathscr{U}$ :

$$
J=\left\{i_{j+1}>i_{j} ; \mu\left(\left\{\left|\phi_{q}\left(i_{1}, \ldots, i_{j}\right)-\phi_{q}\left(i_{1}, \ldots, i_{j+1}\right)\right|<\eta\right\}\right)>1-\eta\right\} .
$$

It follows from (3), (17) that $\phi_{q}$ is a weak cluster point of $\phi_{q}\left(i_{1}, \ldots, i_{j}\right)$ for $\left(i_{1}, \ldots, i_{j}\right)$ in $T$. So (15) shows that we can find a finite set $S$, for $s$ in $S$ numbers $\alpha_{s} \geqslant 0$, with $\sum \boldsymbol{\alpha}_{s}=1$ and elements $\left(i_{1}^{s}, \ldots, i_{j}^{s}\right)$ of $T$ such that

$$
\begin{gathered}
\left\|\sum \alpha_{s} \phi_{q}\left(i_{1}^{s}, \ldots, i_{j}^{s}\right)-\phi_{q}\right\|_{E} \leqslant \varepsilon, \\
\int \xi \psi \phi_{q}\left(i_{1}^{s}, \ldots, i_{j}^{s}\right) d \mu>b^{\prime},
\end{gathered}
$$

where $\xi=\operatorname{sign} \psi \phi$. It follows from (15) that for each $n \in I$, there is $s \in S$ such that

$$
\int_{\left\{\left|\psi_{n}\right|>a\right\}}\left|\psi_{n} \phi_{q}\left(i_{1}^{s}, \ldots, i_{j}^{s}\right)\right| d \mu \leqslant 2 \varepsilon
$$

So, there is $i_{1}<\cdots<i_{j}$ in $T$ such that

$$
\int \xi \psi \phi_{q}\left(i_{1}, \ldots, i_{j}\right) d \mu>b^{\prime}
$$

and $I^{\prime} \in \mathscr{U}$, where

$$
I^{\prime}=\left\{n \in I ; \int_{\left\{\left|\psi_{n}\right|>a\right\}}\left|\psi_{n} \phi_{q}\left(i_{1}, \ldots, i_{j}\right)\right| d \mu \leqslant 2 \varepsilon\right\} .
$$

Since $\psi=$ weak- $\lim _{n \rightarrow \mathscr{U}} \psi_{n}$, we can assume that for $n$ in $I^{\prime}$ we have $\int \xi \psi_{n} \phi_{q}\left(i_{1}, \ldots, i_{j}\right) d \mu>b^{\prime}$. Together with (20), this implies

$$
\int_{\left\{\left|\psi_{n}\right| \leqslant a\right\}} \xi \psi_{n} \phi_{q}\left(i_{1}, \ldots, i_{j}\right) d \mu>b^{\prime}-2 \varepsilon
$$

Since $J \in \mathscr{U}, I^{\prime} \in \mathscr{U}$, we can pick $n \in I^{\prime}$ such that (21) holds and

$$
\mu\left(\left\{\phi_{q}\left(i_{1}, \ldots, i_{j}, n\right)-\phi_{q}\left(i_{1}, \ldots, i_{j+1}\right)<\eta\right\}\right)>1-\eta .
$$

From (18) and (22) follows that

$$
\left\|\phi_{q}\left(i_{1}, \ldots, i_{j}, n\right)-\phi_{q}\left(i_{1}, \ldots, i_{j}\right)\right\|_{1} \leqslant \varepsilon / a,
$$

so we get from (21) that

$$
\int_{\left\{\left|\psi_{n}\right| \leqslant a\right\}} \xi \psi_{n} \phi_{q}\left(i_{1}, \ldots, i_{j}, n\right) d \mu>b^{\prime}-3 \varepsilon
$$


On the other hand, we have

$$
\begin{aligned}
& \left|\int_{\left\{\left|\psi_{n}\right|>a\right\}} \psi_{n} \phi_{q}\left(i_{1}, \ldots, i_{j}, n\right) d \mu\right| \\
& \geqslant\left|\int \psi_{n}\left(\phi_{q}\left(i_{1}, \ldots, i_{j}, n\right)-\phi_{q}\left(i_{1}, \ldots, i_{j}\right)\right) d \mu\right| \\
& \quad-\left|\int_{\left\{\left|\psi_{n}\right| \leqslant a\right\}} \psi_{n}\left(\phi_{q}\left(i_{1}, \ldots, i_{j}, n\right)-\phi_{q}\left(i_{1}, \ldots, i_{j}\right)\right) d \mu\right| \\
& -\left|\int_{\left\{\left|\psi_{n}\right|>a\right\}} \psi_{n} \phi_{q}\left(i_{1}, \ldots, i_{j}\right) d \mu\right| .
\end{aligned}
$$

The first term is $\geqslant 1$ from (5), the second is $\leqslant \varepsilon$ from (18) and (22), and the third is $\leqslant 2 \varepsilon$, since $n \in I^{\prime}$. Finally, we have shown that

$$
\int\left|\psi_{n} \phi_{q}\left(i_{1}, \ldots, i_{j}, n\right)\right| d \mu>1+b^{\prime}-6 \varepsilon>b+1 .
$$

The proof is complete.

\section{REFERENCES}

1. C. D. Aliprantis and O. Burkinshaw, Factoring compact and weakly compact operators through reflexive Banach lattices, Trans. Amer. Math. Soc. 283 (1984), 369-381.

2. W. J. Davis, T. Figiel, W. B. Johnson and A. Pelczynski, Factoring weakly compact operators, J. Funct. Anal. 17 (1974), 311-327.

3. J. Lindenstrauss and L. Tzafriri, Classical Banach spaces. II, Springer-Verlag, New York, 1979.

Department of Mathematics, The Ohio State University, Columbus, Ohio 43210

Equipe d’Analyse, Tour 46, Universite Paris Vi, 4 Place Jussieu, 75230 Paris Cedex 05, France 